\title{
Lactulose Crystals Beneficially Affect Community Composition Along Entire Human Colon in Vitro, Resulting in Donor-Dependent Prebiotic Effects at Metabolic Level
}

\author{
Cindy Duysburgh ${ }^{1}$, Pieter Van den Abbeele ${ }^{1}$, Melanie Bothe ${ }^{2}$, John Stover ${ }^{3}$, \\ Angelika Kuchinka-Koch ${ }^{4}$, Susann Schwejda-Guettes ${ }^{3}$, Massimo Marzorati ${ }^{1,5}$ \\ ${ }^{1}$ Pro Digest Bvba, Ghent, Belgium \\ ${ }^{2}$ Fresenius-Kabi Deutschland GmbH, Bad Homburg, Germany \\ ${ }^{3}$ Fresenius-Kabi Deutschland GmbH, Oberursel, Germany \\ ${ }^{4}$ Fresenius-Kabi Austria GmbH, Linz, Austria \\ ${ }^{5}$ Center of Microbial Ecology and Technology (CMET), Ghent University, Ghent, Belgium
}

Email address:

massimo.marzorati@ugent.be (M. Marzorati)

\section{To cite this article:}

Cindy Duysburgh, Pieter Van Den Abbeele, Melanie Bothe, John Stover, Angelika Kuchinka-Koch, Susann Schwejda-Guettes, Massimo Marzorati. Lactulose Crystals Beneficially Affect Community Composition Along Entire Human Colon in Vitro, Resulting in DonorDependent Prebiotic Effects at Metabolic Level. International Journal of Nutrition and Food Sciences. Vol. 8, No. 1, 2019 , pp. 10-22. doi: $10.11648 /$ j.ijnfs.20190801.12

Received: February 12, 2019; Accepted: March 30, 2019; Published: April 29, 2019

\begin{abstract}
A validated in vitro gut model (i.e. SHIME®) was used to assess the effect of repeated daily administration of lactulose on microbial metabolic activity and community composition in different colonic areas, with the focus on interindividual differences among three human subjects. An initial rise in acetate and lactate levels was observed in the proximal colon after lactulose administration, which could be linked to an overall strong bifidogenic effect as well as higher Lactobacilli levels in donors 2 and 3. Particularly two operational taxonomic units (OTUs) related to Bifidobacterium adolescentis and Bifidobacterium longum increased with lactulose addition. The enhanced acetate and lactate production subsequently stimulated microbial species involved in cross-feeding interactions, resulting in the donor-dependent production of propionate and/or butyrate. Additionally, a reduction in markers of proteolytic fermentation was detected upon lactulose supplementation. A wide spectrum of propionate- and especially butyrate-producing microbes, such as the next-generation probiotics Faecalibacterium prausnitzii and Akkermansia muciniphila, were donor-dependently enhanced in the distal colon, which is of specific interest as many colonic diseases originate in the distal part of the colon. For the first time beneficial effects of lactulose on the microbiota as well as metabolic activity could be demonstrated over the entire colon in vitro.
\end{abstract}

Keywords: Bifidobacteria, Faecalibacterium, Akkermansia, SHIME®, Microbial

\section{Introduction}

The involvement of the human gut microbiome in health and disease has been well established during the past decade. Numerous studies have explored the potential improvement of health by modulating the gut microbiota, mainly by supplementing prebiotics. Prebiotic compounds are classified as non-digestible substrates that are selectively used by the gut microbial community, thereby conferring a health benefit for the host [1]. Lactulose, a synthetic disaccharide composed of $\beta-1,4$-glycosidic bounded galactose and fructose, is an example of such a prebiotic compound. Due to this glycosidic bond, lactulose is resistant to digestion and absorption in the human upper gastro-intestinal tract and reaches the colon unaltered where it can be used as an energy source by the colonic microbiota [2]. Many microbial species have been reported to utilize lactulose, including amongst others several Bifidobacterium and Lactobacillus species [3]. 
Therapeutically, lactulose is widely used for the treatment of constipation [4] and hepatic encephalopathy [5], a syndrome being linked with high ammonium concentrations in the blood due to liver dysfunction. Moreover, lactulose use has been shown to stimulate calcium absorption in animals [6] and postmenopausal women [7]. Other potential health benefits related to lactulose are the stimulation of health-promoting bacteria and the inhibition of pathogenic microorganisms in the gastrointestinal tract [8-10]. For instance, Terada et al. [10] showed that daily ingestion of lactulose by healthy subjects significantly increased bifidobacterial counts in the faecal microbiota, while decreasing the amount of Bacteroidaceae and pathogenic Clostridium species. Cirrhotic patients on the other hand showed higher levels of lactobacilli in stool samples upon repeated lactulose application [11]. Furthermore, De Preter et al. [12] showed that lactulose administration resulted in increased uptake of nitrogen by the colonic microbiota in healthy subjects. Finally, proteolytic metabolites, which have been linked with the onset of several colonic diseases, were reported to be decreased by lactulose in vivo $[9,10]$.

Complementary to in vivo studies, in vitro approaches are widely used to assess the effect of test substances in areas of the gut not easily accessible in vivo. These models allow to work under representative intestinal conditions, ranging from short-term batch incubations [13, 14] to long-term dynamic simulations $[15,16]$. To mimic as close as possible the microbial ecology of the human gastrointestinal tract, in vitro gut models rely on microbial inocula originating from human faecal samples [13]. However, it is widely accepted that the human individuals are highly characterized by interindividual differences in microbial community composition, which can be affected by several aspects such as dietary habits and genetic factors [21]. As these differences in community composition can affect the response to dietary interventions [22], inter-individual variability must be taken into account in in vitro studies. Moreover, the proximal and distal colon are characterized by different microbial communities [23] and there is great interest in identifying compounds able to modulate the activity of the microbiota in the distal colon, an area in which many colonic diseases originate.

Former studies using a computer-controlled in vitro model of the human proximal colon revealed a dosedependent increase in total short-chain fatty acid (SCFA) concentrations upon low-dose lactulose supplementation of up to $5 \mathrm{~g}$ /day, mainly attributed to increased acetate, butyrate and lactate production [17, 18]. Butyrate production, however, was inhibited when higher lactulose amounts (i.e. $10 \mathrm{~g} /$ day) were administered [19]. Furthermore, branched SCFA, $\mathrm{pH}$ and ammonium levels were strongly decreased dose-dependently. The abundance of Bifidobacterium and Lactobacillus species was largely enhanced by lactulose as were several other bacterial species such as Alistipes, Parabacteroides and the butyrate-producing Anaerostipes [17, 20] and
Megasphaera upon supplementation of a liquid and crystalline lactulose formulation, respectively [18]. Altogether, the recent studies of Bothe et al. [17, 18] indicated that a daily dose of $5 \mathrm{~g}$ lactulose was associated with a potent prebiotic effect on the in vitro microbiota of the proximal human colon.

As former in vitro studies have mainly focussed on the effect of lactulose in the human proximal colon, using a pool of human faecal samples as single bacterial inoculum [17, 18], the current work aimed at assessing the impact of repeated daily doses of lactulose crystals on the microbial activity and composition along the entire colon, with a focus on inter-individual differences among three independent human subjects. For this purpose, we made use of the Simulator of the Human Intestinal Microbial Ecosystem $\left(\mathrm{SHIME}^{\circledR}\right)$, a standardized in vitro simulator of the entire human gastrointestinal tract $[24,25]$.

\section{Materials and Methods}

\subsection{Chemicals and Test Product}

All chemicals were obtained from Sigma-Aldrich (Overijse, Belgium) unless stated otherwise. Fresenius-Kabi iPSUM S.r.l. (Vicchio, Italy) provided the lactulose crystals (Ph.Eur.), which were tested at an in vitro dose of 5 grams per day.

\subsection{Simulator of the Human Intestinal Microbial Ecosystem (SHIME $\left.{ }^{\circledR}\right)$}

The reactor configuration was adapted from the SHIME ${ }^{\circledR}$ (ProDigest and Ghent University, Belgium) as described by Molly et al. [16], in order to allow the study of three different donors in one single setup. Each segment of the SHIME consisted of a succession of three reactors simulating the different regions of the gastrointestinal tract, i.e. upper gastrointestinal tract including subsequent simulation of stomach and small intestine, proximal colon (PC) and distal colon (DC), respectively. Inoculum preparation, retention times, $\mathrm{pH}$, temperature settings and reactor feed composition were previously described by Possemiers et al. [26]. Upon inoculation with appropriate fecal samples from three different donors (female, 32y; female, 24y and female, 35y), a two-week stabilization period was initiated to allow the microbial community to differentiate in the different reactors depending on the local environmental conditions, followed by a two-week control period to determine the baseline microbial community composition and activity. Next, $5 \mathrm{~g}$ of the test product was administered once per day during the first feeding cycle over a one-week treatment period. This treatment period was followed by a one-week wash-out phase during which administration of the lactulose crystals was ceased. Finally, the test product was administered daily for another treatment week, with the dosage of $5 \mathrm{~g}$ equally spread over the three feeding cycles during the day. 


\subsection{Microbial Metabolic Activity}

Starting with the two-week control period, samples for microbial metabolic activity were collected three times per week from each colon vessel. Additional samples for metabolic analysis were collected during the second week of control and the final treatment period from each colon compartment at $0,1,2,4,6,8,24,48,72$ and $120 \mathrm{~h}$ after the first addition of the test product. Analysis of SCFA levels, including acetate, propionate, butyrate and branched SCFA (isobutyrate, isovalerate and isocaproate), was performed as described by De Weirdt et al. [27]. Lactate concentrations were determined using a commercially available enzymatic assay kit (R-Biopharm, Darmstadt, Germany) according to manufacturer's instructions. Ammonium analysis was conducted as previously described by Van de Wiele et al. [28].

\subsection{Microbial Community Analysis}

Samples for microbial community analysis were collected once per week from each colon vessel starting from the control phase. DNA was isolated as previously described by Vilchez-Vargas et al. [29], starting from pelleted cells originating from $1 \mathrm{~mL}$ luminal sample.

Subsequently, quantitative polymerase chain reaction (qPCR) for Akkermansia muciniphila, Bifidobacterium spp. and Lactobacillus spp. was performed on a QuantStudio 5 Real-Time PCR system (Applied Biosystems, Foster City, CA USA). Each sample was analysed in technical triplicate and outliers (more than $1 \mathrm{C}_{\mathrm{T}}$ difference) were omitted. The qPCR for Akkermansia muciniphila was performed using the protocol as described by Collado et al. [30]. The qPCR for Lactobacillus and Bifidobacterium spp. were conducted as described by Furet et al. [31] and Rinttilä et al. [32], respectively.

The microbiota profiling of each colon compartment was established by $16 \mathrm{~S}$-targeted Illumina sequencing analysis. The 16S rRNA gene V3-V4 hypervariable regions were amplified by PCR using primers 341F (5'-CCT ACG GGN GGC WGC AG -3') and 785Rmod (5'-GAC TAC HVG GGT ATC TAA KCC-3'), adapted from Klindworth et al. [33]. The original genomic DNA extracts were diluted in DNase/RNase/protease-free water to obtain a concentration of $50 \mathrm{ng} / \mu \mathrm{L}$ and $30 \mu \mathrm{L}$ was send out to LGC genomics $\mathrm{GmbH}$ (Germany) for library preparation and sequencing on an Illumina Miseq platform with v3 chemistry with the primers mentioned above.

\subsection{Statistics}

Comparison of normally distributed data of the different experimental periods on microbial metabolic markers and microbial community parameters was performed with a Student's T-test for pairwise comparisons. Differences were considered significant if $\mathrm{p}<0.05$.

For the 16S-targeted sequencing analysis, read assembly and cleanup was derived from the MiSeq protocol described by the Schloss lab [34, 35]. In brief, mothur (v. 1.39.5) was used to assemble reads into contigs, perform alignment-based quality filtering (alignment to the mothur-reconstructed SILVA SEED alignment, v. 123), remove chimeras, assign taxonomy using a naïve Bayesian classifier [36] and SILVA NR v128 and cluster contigs into OTUs at 97\% sequence similarity. All sequences classified as Eukaryota, Archaea, Chloroplasts and Mitochondria were removed, as well as sequences that could not be classified. For each OTU, representative sequences were selected as the most abundant sequence within that OTU.

\section{Results}

\subsection{Analysis of the Microbial Metabolic Activity}

The SCFA profiles mainly consisted of acetate, propionate and butyrate (Table 1) and small concentrations of branched SCFA (Table 2). Lactulose crystals significantly enhanced acetate levels compared to the control period in all colon regions, except in the $\mathrm{PC}$ of donor 2. The strongest effect was detected for donor 3 during the first treatment week with an average increase in acetate production of $24.7 \mathrm{mM}(+99.8 \%)$ and $37.9 \mathrm{mM}(+70.3 \%)$ in the $\mathrm{PC}$ and $\mathrm{DC}$, respectively. Propionate levels were increased in both colon regions of donor 1 by lactulose. On the other hand, administration of crystalline lactulose to the colon microbiota of donor 2 and 3 resulted in a statistically significant reduction of propionate levels compared to the control period, especially during the final week of treatment. For donor 1, butyrate levels remained very low and, therefore, unaffected by lactulose. For donor 2 and 3, butyrate levels significantly rised upon addition of lactulose in both the PC and DC compared to the control period. This effect was most pronounced in the PC of donor 2 with significant increases of $24.2 \mathrm{mM}(+200.1 \%)$ and $25.2 \mathrm{mM}(+208.4 \%)$ during the first and second treatment period, respectively.

Table 1. Overall Metabolic Activity In Terms Of SCFA And Lactate Production.

\begin{tabular}{lllllll}
\hline & & C & TR1 & WO & \multicolumn{1}{l}{ TR2 } \\
\hline & \multirow{3}{*}{ PC } & Donor 1 & $23.0( \pm 1.3)$ & $31.2( \pm 2.2)$ & $23.1( \pm 4.0)$ & $36.1( \pm 6.3)$ \\
Acetate $(\mathrm{mM})$ & Donor 2 & $21.5( \pm 0.9)$ & $30.4( \pm 19.4)$ & $20.0( \pm 2.3)$ & $23.7( \pm 4.0)$ \\
& & Donor 3 & $24.7( \pm 4.1)$ & $49.4( \pm 10.6)$ & $23.8( \pm 12.3)$ & $35.4( \pm 6.1)$ \\
& \multirow{2}{*}{ DC } & Donor 1 & $58.0( \pm 3.6)$ & $71.3( \pm 10.4)$ & $50.9( \pm 6.9)$ & $51.4( \pm 8.5)$ \\
\multirow{2}{*}{ Propionate $(\mathrm{mM})$} & & Donor 2 & $55.5( \pm 2.7)$ & $78.8( \pm 14.1)$ & $50.4( \pm 10.9)$ & $46.6( \pm 7.5)$ \\
& & Donor 3 & $53.9( \pm 2.0)$ & $91.9( \pm 19.4)$ & $48.7( \pm 14.9)$ & $53.0( \pm 17.3)$ \\
& \multirow{2}{*}{ PC } & Donor 1 & $30.3( \pm 1.6)$ & $70.2( \pm 3.2)$ & $31.1( \pm 9.4)$ & $66.2( \pm 19.1)$ \\
\hline
\end{tabular}




\begin{tabular}{lllllll}
\hline & & C & TR1 & WO & TR2 \\
\hline & & Donor 3 & $15.8( \pm 1.1)$ & $9.1( \pm 5.3)$ & $14.6( \pm 5.2)$ & $7.2( \pm 4.0)$ \\
& & Donor 1 & $50.3( \pm 3.3)$ & $88.2( \pm 24.9)$ & $61.0( \pm 21.6)$ & $71.9( \pm 26.7)$ \\
& DC & Donor 2 & $22.2( \pm 1.9)$ & $22.9( \pm 2.5)$ & $19.7( \pm 4.8)$ & $14.5( \pm 1.0)$ \\
& & Donor 3 & $22.3( \pm 0.9)$ & $21.4( \pm 0.8)$ & $22.1( \pm 6.3)$ & $14.7( \pm 1.8)$ \\
& & Donor 1 & $0.10( \pm 0.05)$ & $0.26( \pm 0.12)$ & $0.03( \pm 0.05)$ & $0.04( \pm 0.07)$ \\
Butyrate $(\mathrm{mM})$ & PC & Donor 2 & $12.1( \pm 1.5)$ & $36.2( \pm 11.0)$ & $19.7( \pm 6.0)$ & $37.3( \pm 17.2)$ \\
& & Donor 3 & $10.4( \pm 1.3)$ & $23.9( \pm 8.4)$ & $10.4( \pm 2.5)$ & $26.3( \pm 9.9)$ \\
& & Donor 1 & $7.09( \pm 0.39)$ & $8.87( \pm 1.17)$ & $7.23( \pm 1.18)$ & $8.07( \pm 1.77)$ \\
& DC & Donor 2 & $17.6( \pm 1.0)$ & $37.6( \pm 14.2)$ & $30.8( \pm 12.2)$ & $37.3( \pm 19.5)$ \\
& & Donor 3 & $16.6( \pm 0.9)$ & $29.6( \pm 9.7)$ & $15.9( \pm 2.2)$ & $27.6( \pm 11.6)$ \\
& & Donor 1 & $0.10( \pm 0.06)$ & $1.88( \pm 0.05)$ & $0.23( \pm 0.13)$ & $0.20( \pm 0.18)$ \\
& PC & Donor 2 & $0.30( \pm 0.33)$ & $4.46( \pm 5.12)$ & $1.09( \pm 0.69)$ & $4.70( \pm 5.88)$ \\
& & Donor 3 & $0.29( \pm 0.31)$ & $3.42( \pm 2.98)$ & $0.20( \pm 0.16)$ & $2.18( \pm 1.51)$ \\
& & Donor 1 & $0.41( \pm 0.20)$ & $0.51( \pm 0.03)$ & $0.64( \pm 0.02)$ & $0.31( \pm 0.05)$ \\
& DC & Donor 2 & $0.58( \pm 0.26)$ & $1.01( \pm 0.09)$ & $1.18( \pm 0.47)$ & $1.28( \pm 0.39)$ \\
& & Donor 3 & $0.42( \pm 0.03)$ & $0.82( \pm 0.12)$ & $0.61( \pm 0.22)$ & $0.54( \pm 0.14)$ \\
\hline
\end{tabular}

Average acetate, propionate, butyrate and lactate production $(\mathrm{mM})$ during the control $(\mathrm{C} ; \mathrm{n}=6)$, the wash-out $(\mathrm{WO} ; \mathrm{n}=3)$ and the two treatment $(\mathrm{TR} 1 / 2 ; \mathrm{n}=$ 3 ) weeks in the proximal (PC) and distal colon (DC) of the human gastro-intestinal tract for three donors tested upon treatment with the test product. Data is presented as mean \pm stdev. Statistically significant differences relative to the control period are indicated in bold $(\mathrm{p}<0.05)$.

Overall, branched SCFA levels were low (Table 2), with a significantly reduced production observed during the second application week in both colon regions for all donors tested, except for donor 1 in the PC where branched SCFA levels remained below detection limit. Examination of average weekly ammonium production showed no effect in both colon regions, except for a significant decrease in the DC during the final week of treatment for donor 2 (Table 2). However, when daily levels were compared during the second week of administration and measurement after $0,1,2$,
4, 6, 8, 24, 48, 72 and 120h (Figure A1), a trend towards reduced ammonium levels was observed for all donors tested. Finally, in the PC, lactate concentrations were significantly elevated during the first week of administration with the test product for the three donors. For donor 2 and 3, higher lactate levels were also detected during the final treatment week. In the DC, slightly increased lactate concentrations were seen after supplementation of lactulose for donor 2 and 3.

Table 2. Microbial Metabolic Activity In Terms Of Branched SCFA And Ammonium Production.

\begin{tabular}{|c|c|c|c|c|c|c|}
\hline & & & C & TR1 & Wo & TR2 \\
\hline \multirow{6}{*}{ Branched SCFA (mM) } & \multirow{3}{*}{$\mathrm{PC}$} & Donor 1 & $0.00( \pm 0.00)$ & $0.07( \pm 0.06)$ & $0.00( \pm 0.00)$ & $0.00( \pm 0.00)$ \\
\hline & & Donor 2 & $2.95( \pm 0.14)$ & $2.63( \pm 0.36)$ & $2.34( \pm 0.18)$ & $1.77( \pm 0.38)$ \\
\hline & & Donor 3 & $3.00( \pm 0.13)$ & $2.81( \pm 0.56)$ & $2.45( \pm 0.48)$ & $2.11( \pm 0.32)$ \\
\hline & \multirow{3}{*}{ DC } & Donor 1 & $2.52( \pm 0.11)$ & $2.22( \pm 0.25)$ & $2.22( \pm 0.08)$ & $1.99( \pm 0.15)$ \\
\hline & & Donor 2 & $3.35( \pm 0.12)$ & $3.03( \pm 0.35)$ & $2.85( \pm 0.06)$ & $2.36( \pm 0.37)$ \\
\hline & & Donor 3 & $3.27( \pm 0.17)$ & $3.04( \pm 0.42)$ & $2.53( \pm 0.25)$ & $2.26( \pm 0.16)$ \\
\hline \multirow{6}{*}{ Ammonium (mg/L) } & \multirow{3}{*}{$\mathrm{PC}$} & Donor 1 & $36( \pm 25)$ & $27( \pm 4)$ & $62( \pm 19)$ & $39( \pm 10)$ \\
\hline & & Donor 2 & $191( \pm 78)$ & $133( \pm 28)$ & $176( \pm 28)$ & $200( \pm 46)$ \\
\hline & & Donor 3 & $139( \pm 44)$ & $135( \pm 31)$ & $161( \pm 42)$ & $147( \pm 42)$ \\
\hline & \multirow{3}{*}{ DC } & Donor 1 & $217( \pm 66)$ & $207( \pm 51)$ & $232( \pm 58)$ & $220( \pm 34)$ \\
\hline & & Donor 2 & $253( \pm 51)$ & $233( \pm 71)$ & $161( \pm 39)$ & $146( \pm 33)$ \\
\hline & & Donor 3 & $243( \pm 49)$ & $214( \pm 66)$ & $252( \pm 27)$ & $247( \pm 80)$ \\
\hline
\end{tabular}

Average branched SCFA $(\mathrm{mM})$ and ammonium $(\mathrm{mg} / \mathrm{L})$ production during the control $(\mathrm{C} ; \mathrm{n}=6)$, the wash-out $(\mathrm{WO} ; \mathrm{n}=3)$ and the two treatment $(\mathrm{TR} 1 / 2 ; \mathrm{n}=$ 3 ) weeks in the proximal (PC) and distal colon (DC) of the human gastro-intestinal tract for three donors tested upon treatment with the test product. Data is presented as mean \pm stdev. Statistically significant differences relative to the control period are indicated in bold $(\mathrm{p}<0.05)$.

The SCFA profiles also revealed a distinct effect in the production of different metabolites upon lactulose addition over time (Figure 1). In the PC (and to a lesser extent in the DC) of donor 2, an immediate rise in acetate and lactate concentrations was observed during the first treatment period, followed by a decrease of acetate levels at the expense of butyrate production. At the end of the first week of administration, butyrate levels were slightly reduced at the expense of higher propionate levels. During the wash-out phase, SCFA levels decreased reaching similar levels compared to the control period. Finally, during the second application week, mainly elevated amounts of butyrate were seen. Similar observations were made for donor 3, whereas donor 1 mainly showed strongly increased propionate production following supplementation of lactulose. 
A

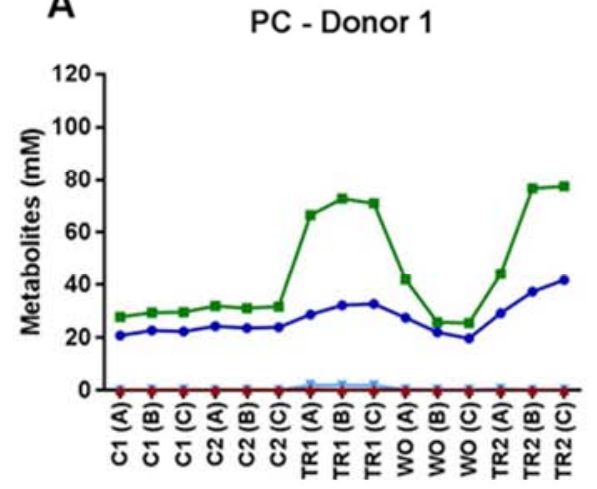

C

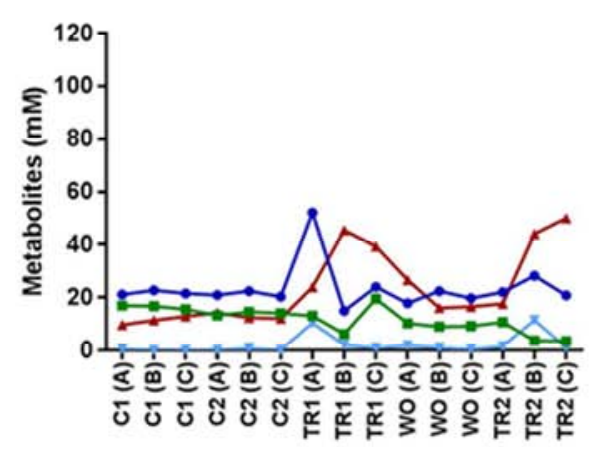

E

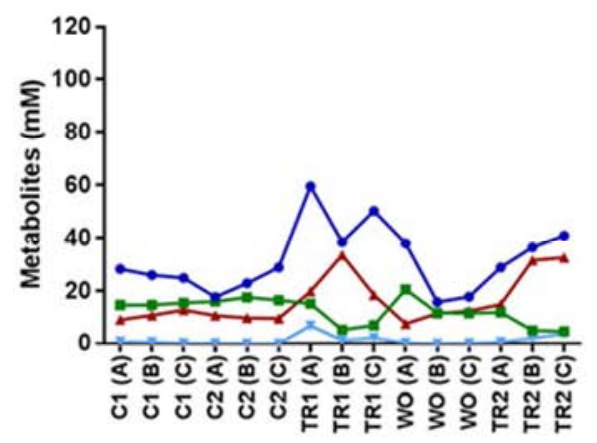

B
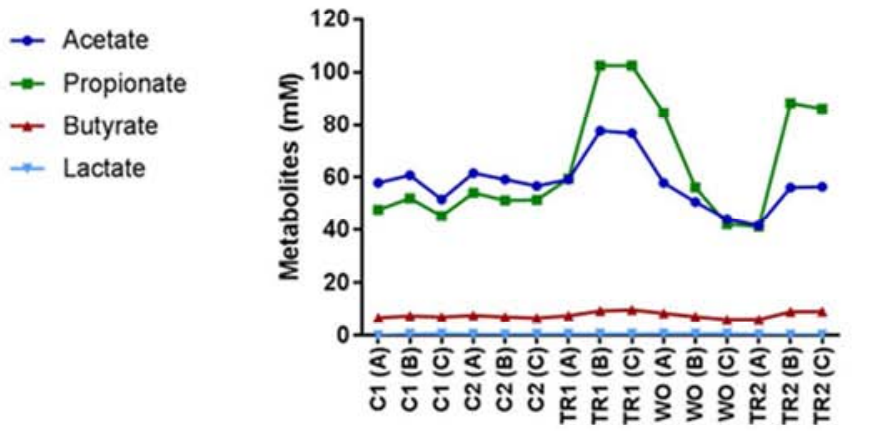

$\rightarrow$ Acetate
- Propionate
+ Butyrate
$\rightarrow$ Lactate

D

DC - Donor 2
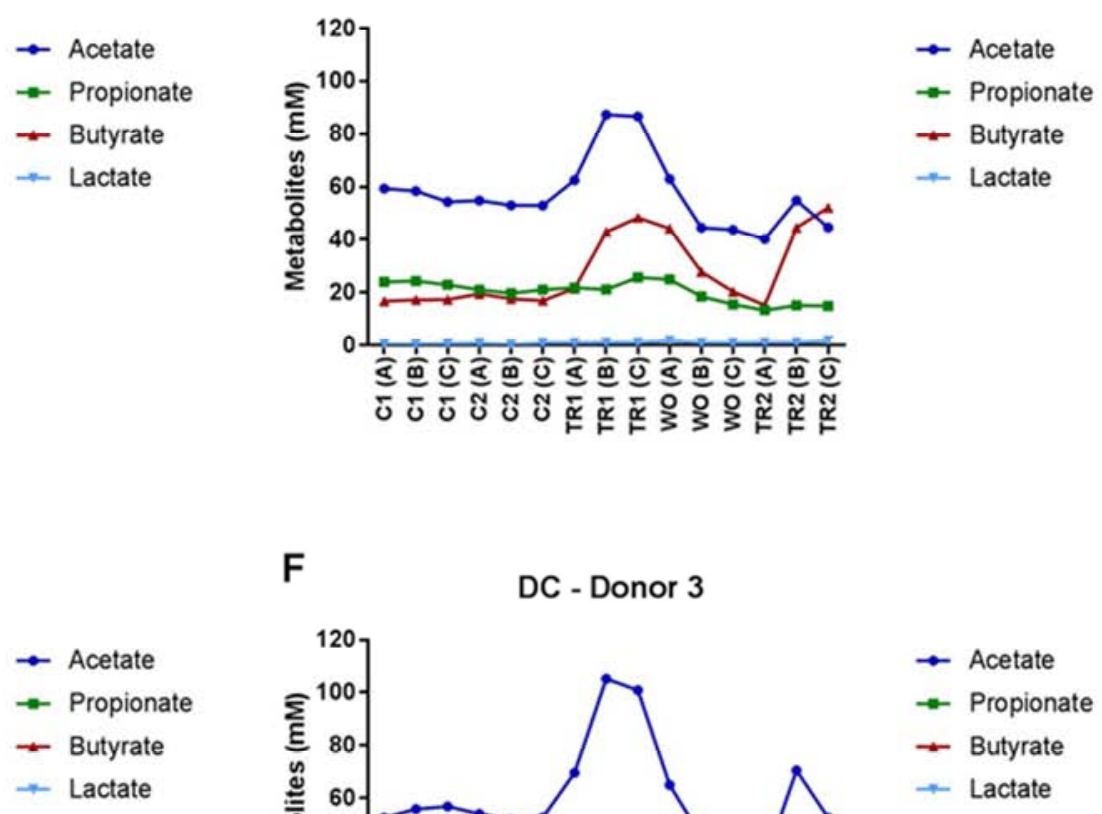

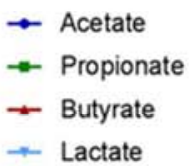

$\mathbf{F}$

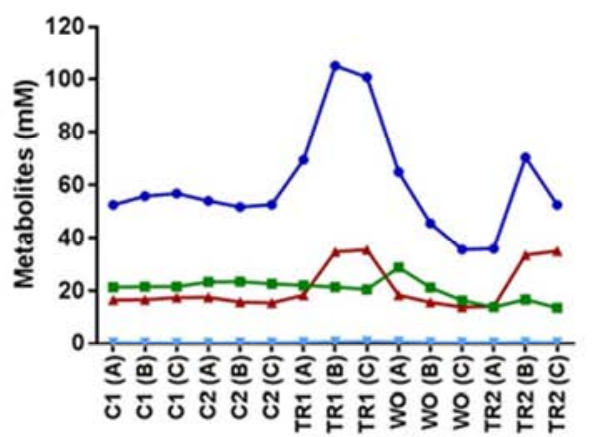

Figure 1. Microbial Metabolic Activity Over Time.

Absolute concentrations ( $\mathrm{mM}$ ) of acetate, propionate, butyrate and lactate associated with supplementation of the test product to the colon microbiota of three human donors in the proximal (PC) and distal colon (DC) compartment. Samples were taken during two control (C1 and C2), one wash-out (WO) and two treatment (TR1 and TR2) weeks. During each week, three samples (A, B and C) were collected.

\subsection{Analysis of the Microbial Community Composition}

The main phyla in the microbial community of the three donors prior to the experimental phase with lactulose included Actinobacteria, Bacteroidetes, Firmicutes, Fusobacteria, Proteobacteria and Verrucomicrobia, showing specific colonization patterns in the different colonic regions (Figure 2). Verrucomicrobia and Fusobacteria specifically colonized the DC area, which was also observed for the Ruminococcaceae family within the Firmicutes phylum.
Furthermore, several families of the Bacteroidetes phylum specifically inhabited the DC. Families within the Actinobacteria phylum, e.g. Bifidobacteriaceae and Microbacteriaceae, on the other hand were primarily present in the PC, as were several families within the Proteobacteria phylum. Finally, the microbial community composition showed that donor 1 was characterized by a lower species richness and evenness as compared to the other donors, as seen by the high abundance of the Veillonellaceae family, mainly in the PC. 


\begin{tabular}{|c|c|c|c|c|c|c|c|}
\hline \multirow{3}{*}{ Phylum } & \multirow{3}{*}{ Family } & \multicolumn{6}{|c|}{ Control } \\
\hline & & \multicolumn{2}{|c|}{ Donor 1 } & \multicolumn{2}{|c|}{ Donor 2} & \multicolumn{2}{|c|}{ Donor 3} \\
\hline & & PC & DC & PC & DC & PC & DC \\
\hline \multirow{2}{*}{ Actinobacteria } & Bifidobacteriaceae & - & 0.1 & 1.5 & 1.2 & 1.8 & 0.6 \\
\hline & Microbacteriaceae & 0.2 & - & 0.1 & - & 0.5 & 0.1 \\
\hline \multirow{6}{*}{ Bacteroidetes } & Bacteroidaceae & 0.9 & 13.1 & 54.7 & 38.7 & 64.7 & 34.5 \\
\hline & Muribaculaceae & - & 2.9 & - & 0.8 & - & - \\
\hline & Prevotellaceae & - & - & - & 2.3 & - & 1.2 \\
\hline & Rikenellaceae & - & - & - & 0.2 & - & 0.2 \\
\hline & Tannerellaceae & - & 1.3 & - & 5.5 & - & 6.5 \\
\hline & Acidaminococcaceae & - & 1.5 & 5.1 & 2.5 & 4.7 & 2.3 \\
\hline \multirow{4}{*}{ Fimicutes } & Lachnospiraceae & 4.6 & 2.9 & 33.5 & 36.8 & 23.2 & 34.6 \\
\hline & Lactobacillaceae & - & - & 0.3 & 0.1 & - & - \\
\hline & Ruminococcaceae & - & 0.1 & - & 1.5 & - & 2.5 \\
\hline & Veillonellaceae & 93.7 & 56.6 & 1.2 & 1.5 & 0.2 & 1.1 \\
\hline \multirow[t]{2}{*}{ Fusobacteria } & Fusobacteriaceae & - & 0.1 & - & 5.0 & - & 7.5 \\
\hline & Burkholderiaceae & 0.1 & 0.2 & 1.3 & 1.0 & 1.6 & 1.4 \\
\hline \multirow{5}{*}{ Proteobacteria } & Desulfovibrionaceae & - & 0.3 & 1.8 & 1.2 & 1.9 & 1.2 \\
\hline & Enterobacteriaceae & - & - & 0.1 & $\cdot$ & - & - \\
\hline & Pseudomonadaceae & 0.1 & 0.2 & 0.2 & 0.9 & 1.2 & 1.5 \\
\hline & uncultured & - & 0.2 & - & 0.1 & - & 4.4 \\
\hline & Xanthomonadaceae & 0.4 & - & 0.1 & - & 0.2 & 0.1 \\
\hline Verrucomicrobia & Akkermansiaceae & - & 20.2 & - & 0.4 & - & 0.2 \\
\hline
\end{tabular}

Figure 2. Colon-Region Specific Colonization of Microbial Community

Average abundance (\%) at microbial family level in the proximal (PC) and distal colon (DC) of the Simulator of the Human Intestinal Microbial Ecosystem (SHIME $®)$ at the end of the control period for three different human donors $(\mathrm{n}=1)$. The intensity of green shading indicates the absolute abundance, normalized for each of the different families. Lowest values within each family are indicated with two shades of red, medium values are indicated in white, whereas the highest values within each family are indicated with two shades of green.

Upon application of lactulose crystals, a rise in the Actinobacteria phylum was detected in both colon regions, with the effect being less pronounced for donor 1 compared to donor 2 and 3 (Figure 3). In the PC, Actinobacteria levels were elevated at the expense of Bacteroidetes, whereas in the DC the abundance of the Actinobacteria phylum enhanced at the expense of Firmicutes. The strongest effects were seen during the final week of treatment. During that period, an additional boost in Proteobacteria levels was observed in the PC for all three donors tested, whereas in the DC interindividual differences were perceived after administration of the test product (i.e. Proteobacteria levels remained unaffected for donor 1 , increased for donor 2 and even decreased for donor 3). Verrucomicrobia levels did not change in all tested microbiota samples after addition of lactulose. However, a bloom of Verrucomicrobia was observed during the wash-out period for donor 2 in the DC. At family level (Figure A2 and A3), the consistent growth enhancement of the Actinobacteria phylum was mainly attributed to an increased abundance of Bifidobacteriaceae. At the lowest phylogenetic level (OTU level; Figure 4) the higher abundance of Bifidobacteriaceae in PC and DC was mainly related to a rise in Bifidobacterium adolescentis (OTU 7) and Bifidobacterium longum (OTU 16). In the PC, donor-specific changes were detected for donor 2 after lactulose treatment including higher Lactobacillaceae counts and a specific strong enhancement in Veillonellaceae OTU 12 related to Megasphaera sp. during both supplementation periods. In the DC, supplementation of crystalline lactulose resulted in increased abundance of Rikenellaceae for donor 1 and 3, especially during the final week of treatment, whereas donor 2 was characterized by a strong growth of Tannerellaceae. At OTU level, these changes were found to be attributed to a bloom in Rikenellaceae OTU 33 (related to Alistipes onderdonkii) and Tannerellaceae OTU 10 (related to Parabacteroides distasonis) and OTU 24 (related to Parabacteroides sp.). Furthermore, in the DC the total abundance of the butyrate-producing Lachnospiraceae and Ruminococcaceae (both members of the Firmicutes phylum) overall remained similar between the first week of administration and the control period, while increasing during the second application phase versus the wash-out period with $2.1 \%, 9.7 \%$ and $8.7 \%$ for donor 1,2 and 3 , respectively. For donor 2, OTU 47 related to Faecalibacterium prausnitzii raised 48-fold accounting for an increase of $2.0 \%$. Finally, the bloom of Verrucomicrobia in the DC that was noticed during the wash-out phase for donor 2, was linked to a specific rise in OTU 5 related to Akkermansia muciniphila. 

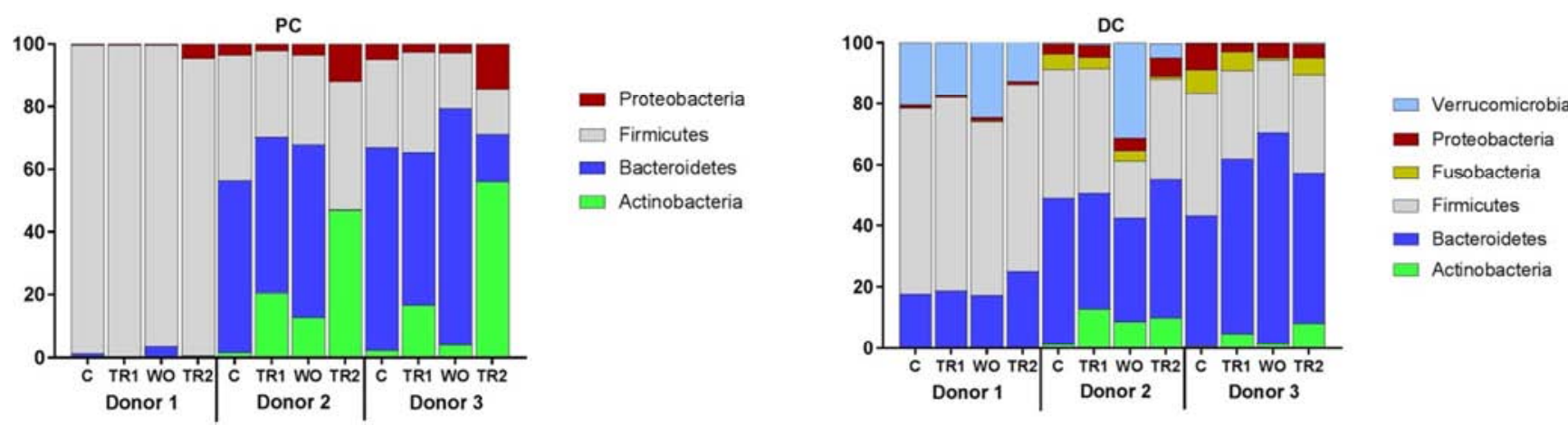

Figure 3. Microbial Community Composition As Assessed Via 16S-targeted Illumina Sequencing.

Abundance (\%) at microbial phylum level in the proximal (PC) and distal colon (DC) of the human gastro-intestinal tract at the end of the control (C), washout (WO) and the two treatment (TR1/2) periods upon treatment with the test product for three donors tested $(\mathrm{n}=1)$.

\begin{tabular}{|c|c|c|c|c|c|c|c|}
\hline \multirow{2}{*}{$\begin{array}{l}\text { Colon } \\
\text { region }\end{array}$} & \multirow{2}{*}{ Phylum } & \multirow{2}{*}{ Family } & \multirow{2}{*}{ OTU } & \multicolumn{4}{|c|}{ Donor 1} \\
\hline & & & & C & TRI & wo & TR2 \\
\hline \multirow{4}{*}{ PC } & \multirow{2}{*}{ Actinobacteria } & \multirow{2}{*}{ Bifidobacteriaceae } & OTU 7 - Bifidobacterizm adolescentis & - & - & - & - \\
\hline & & & OTU 16 - Bifidobacterizm longum & - & - & 0.1 & - \\
\hline & \multirow{2}{*}{ Firmicutes } & Veillonellaceae & OTU 12 -Megasphaerasp. & - & - & $\cdot$ & - \\
\hline & & Lactobacillaceae & OTU 46 - Lactobacillus buchneri & - & - & - & - \\
\hline \multirow{6}{*}{ DC } & \multirow{3}{*}{ Bacteroidetes } & \multirow{2}{*}{ Tannerellaceae } & OTU 10 - Parabacteroides distasonis & 0.7 & 0.5 & 2.9 & 0.1 \\
\hline & & & OTU 24 - Parabacteroides sp. & 0.2 & 0.1 & 0.4 & 0.2 \\
\hline & & Rikenellaceae & OTU 33 - Alistipes onderdonkii & - & 0.7 & 0.1 & 1.1 \\
\hline & Fimicutes & \multicolumn{2}{|c|}{ Lachnospiraceae + Ruminococcaceae } & 3.1 & 4.6 & 4.0 & 6.1 \\
\hline & Fimicutes & Ruminococcaceae & OTU 47 - Faecalibacterium prausnitzii & - & - & - & 0.5 \\
\hline & Verrucomicrobia & Akkermansiaceae & OTU 5 - Akkermansia muciniphila & 20.1 & 17.2 & 24.4 & 12.7 \\
\hline \multirow{2}{*}{$\begin{array}{l}\text { Colon } \\
\text { region }\end{array}$} & \multirow{2}{*}{ Phylum } & \multirow{2}{*}{ Family } & \multirow{2}{*}{ OTU } & \multicolumn{4}{|c|}{ Donor 2} \\
\hline & & & & C & TRl & Wo & TR2 \\
\hline \multirow{4}{*}{ PC } & \multirow{2}{*}{ Actinobacteria } & \multirow{2}{*}{ Bifidobacteriaceae } & OTU 7 - Bifidobacteritom adolescentis & 1.5 & 20.5 & 12.7 & 45.2 \\
\hline & & & OTU 16 - Bifidobacterizm longum & - & 0.1 & 0.1 & 0.4 \\
\hline & \multirow{2}{*}{ Firmicutes } & Veillonellaceae & OTU 12 - Megasphaera sp. & 0.5 & 8.3 & 1.7 & 39.2 \\
\hline & & Lactobacillaceae & OTU 46 - Lactobacillus buchneri & 0.3 & 1.9 & 0.8 & 1.0 \\
\hline \multirow{6}{*}{ DC } & \multirow{3}{*}{ Bacteroidetes } & \multirow{2}{*}{ Tannerellaceae } & OTU 10 - Parabacteroides distasonis & 4.1 & 5.3 & 7.7 & 8.0 \\
\hline & & & OTU 24 - Parabacteroides sp. & 1.4 & 2.7 & 0.8 & 2.3 \\
\hline & & Rikenellaceae & OTU 33 - Alistipes onderdonkii & - & 0.3 & - & 0.1 \\
\hline & & \multicolumn{2}{|c|}{ Lachnospiraceae + Ruminococcaceae } & 38.4 & 37.3 & 15.9 & 25.7 \\
\hline & Firmicutes & Ruminococcaceae & OTU 47 - Faecalibacterium prausnitzii & 0.6 & 0.4 & - & 2.0 \\
\hline & Verrucomicrobia & Akkermansiaceae & OTU 5 -Akkermansia muciniphila & 0.4 & 0.3 & 31.1 & 4.8 \\
\hline \multirow{2}{*}{$\begin{array}{l}\text { Colon } \\
\text { region }\end{array}$} & & & & Donc & & & \\
\hline & Phylum & Family & Оте & C & TRI & wo & TR2 \\
\hline & & & OTU 7 - Bifidobacterizm adolescentis & 0.5 & 5.2 & 2.3 & 38.9 \\
\hline & Actinobacteria & Bifidobacteriaceae & OTU 16 - Bifidobacterizom longum & 1.3 & 11.3 & 1.7 & 13.3 \\
\hline $\mathrm{PC}$ & Fimicutes & Veillonellaceae & OTU 12 - Megasphaerasp. & - & - & - & - \\
\hline & Fimmicutes & Lactobacillaceae & OTU 46 - Lactobacillus buchneri & - & - & - & - \\
\hline & & Tannerellaceae & OTU 10 - Parabacteroides distasonis & 3.9 & 2.8 & 2.8 & 3.3 \\
\hline & Bacteroidetes & Tannerellaceae & OTU 24 - Parabacteroides sp. & 1.8 & 1.0 & 2.6 & 0.7 \\
\hline & & Rikenellaceae & OTU 33 - Alistipes onderdonkii & - & 0.8 & 1.4 & 4.2 \\
\hline DC & & Lachnospiraceae + & occaceae & 37.0 & 27.7 & 21.1 & 29.8 \\
\hline & & Ruminococcaceae & OTU 47 - Faecalibacterium prausnitzii & - & - & - & - \\
\hline & Vemucomicrobia & Akkermansiaceae & OTU 5 -Akkermansia muciniphila & 0.2 & 0.1 & 0.1 & 0.4 \\
\hline
\end{tabular}

Figure 4. Microbial Community Composition As Assessed Via 16S-targeted Illumina Sequencing at OTU Level.

Abundance (\%) of specific microbial OTUs in the proximal (PC) and distal colon (DC) of the Simulator of the Human Intestinal Microbial Ecosystem (SHIME®) at the end of the control (C), the wash-out (WO) and the two treatment (TR1/2) periods for three different human donors $(\mathrm{n}=1)$. The intensity of shading indicates the absolute abundance, normalized for each of the different OTUs. Lowest values within each donor are indicated with two shades of red, medium values are indicated in white, whereas the highest values within each donor are indicated with two shades of green.

To assess the effect of lactulose on specific taxonomic groups of interest (Bifidobacterium spp., Lactobacillus spp. and Akkermansia muciniphila), qPCR analysis was performed (Figure 5). In the PC, a significant stimulation of bifidobacteria levels was seen. For donor 1 , bifidobacteria levels even elevated from below detection limit to $8.0 * 10^{6}$ 
16S rRNA copies/mL. Furthermore, lactobacilli levels increased upon administration of the test product, however only in the PC of donor 2 and 3. Finally, qPCR analysis confirmed the observation of higher Akkermansia muciniphila counts during the wash-out period for donor 2, while no significant effects were detected after addition of crystalline lactulose.
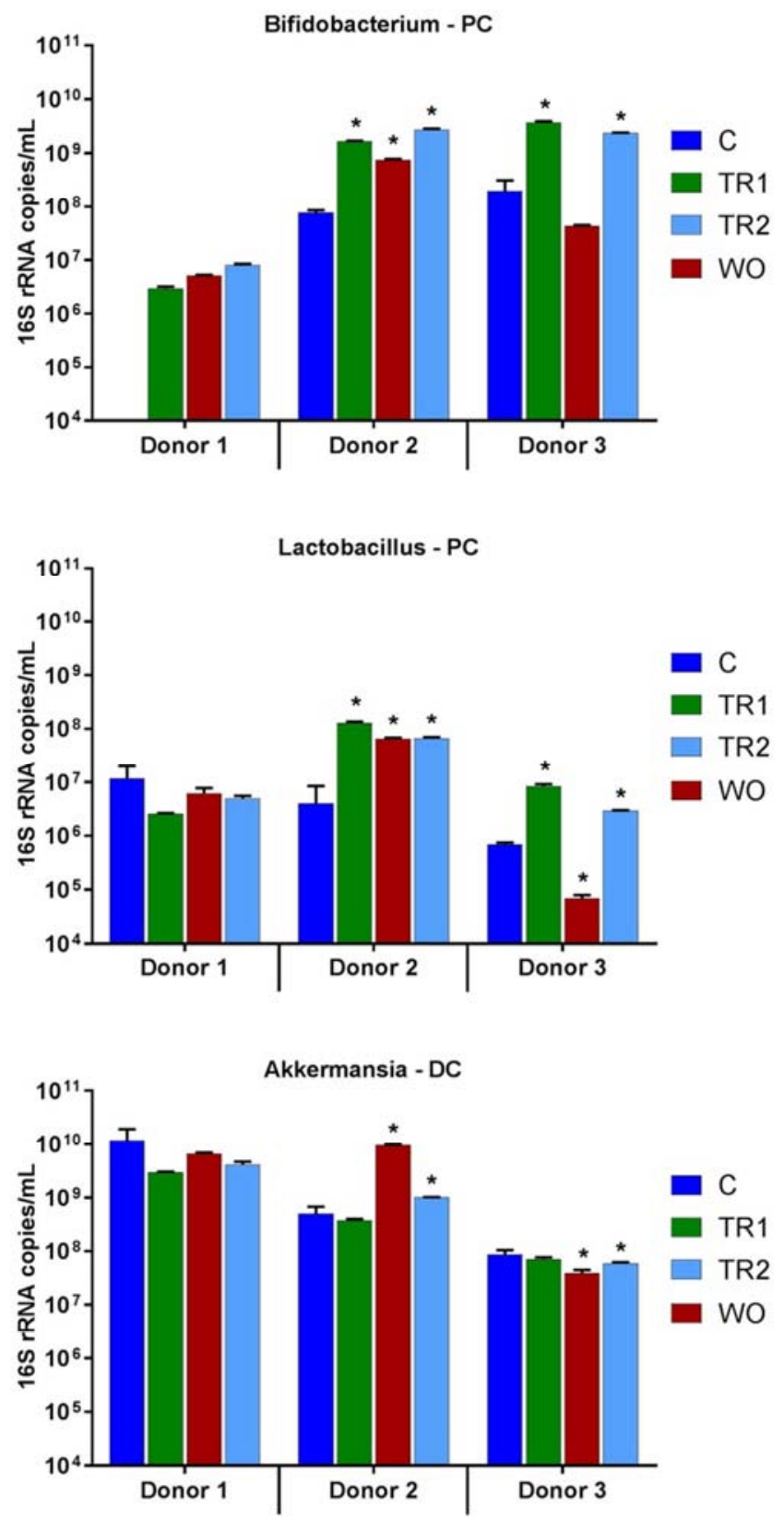

Figure 5. Microbial Community Composition As Assessed Via qPCR.

Average Bifidobacterium spp., Lactobacillus spp. and Akkermansia muciniphila levels (16S rRNA copies $/ \mathrm{mL})$ over the control $(\mathrm{C} ; \mathrm{n}=6)$, the wash-out (WO; $n=3)$ and the two treatment $(\mathrm{TR} 1 / 2 ; n=3)$ periods in the proximal (PC) or distal colon (DC) of the human gastro-intestinal tract for three donors upon treatment with the test product. Data is presented as mean \pm stdev. Statistically significant differences relative to the control period are indicated with $*(\mathrm{p}<0.05)$.

\section{Discussion}

In the present study, the effect of repeated daily administration of $5 \mathrm{~g}$ lactulose crystals on the microbial metabolic activity and community composition in the human gastro-intestinal tract was assessed over a period of two intermittent weeks, taking into account the issue of inter-individual variability. For this purpose, the validated in vitro Simulator of the Human Intestinal Microbial Ecosystem (SHIME) was used. Microbial community development was shown to be colon-region specific. The PC was characterized by increased abundance of Actinobacteria, while the DC displayed higher abundance of Bacteroidetes and Ruminococcaceae and a specific colonization of members of the Verrucomicrobia phylum (i.e. Akkermansia muciniphila). These observations corresponded with the findings of Van den Abbeele et al. [23], which showed a colon-specific colonization of the gut microbiota. This results from the imposed conditions that favour a saccharolytic microbial community in the PC while selecting for species with specific metabolic functions, such as mucin degradation, in the DC [23]. The propionate-producing, mucin-degrading Akkermansia muciniphila for instance has recently been shown to specifically colonize DC areas, while being virtually absent in the PC [37].

Overall, lactulose induced an initial increase in acetate and lactate levels, which could be linked with a strong bifidogenic effect that was observed in the PC for all tested microbial communities of healthy individuals. Particularly two OTUs related to Bifidobacterium adolescentis and Bifidobacterium longum were elevated after lactulose administration in both, PC and DC. Additionally, also the lactate-producing actobacilli showed enhanced growth for donor 2 and 3 in the PC. Lactobacilli and bifidobacteria are considered as beneficial saccharolytic microorganisms, capable of producing high levels of acetate and lactate [38]. These metabolites are important in the human colonic environment as they are the driver of a series of trophic interactions with other bacteria, resulting in the production of downstream metabolites, such as propionate and butyrate [39]. Furthermore, lactate is known to exert strong antimicrobial effects against pathogenic species [40]. Similar studies performed by Bothe et al. [17, 18] showed that daily administration of lactulose, both as liquid or crystalline formulation, enhanced specific growth of Bifidobacterium and Lactobacillus species, resulting in a predominant increase in acetate levels in vitro. Furthermore, several studies have shown an enhanced growth in faecal Bifidobacterium following uptake of lactulose in vivo [41-43]. For instance, Vanhoutte et al. [43] showed specifically elevated Bifidobacterium adolescentis levels in faecal samples of healthy human subjects after repeated lactulose intake, thereby confirming the specific observations in the present in vitro study. 
The initial rise in acetate and lactate production after lactulose intake was followed by an efficient conversion to propionate and/or butyrate in a donor-dependent manner. Whereas the microbiota of donor 1 specifically supported propionate production upon lactulose supplementation, the microbial communities of donor 2 and 3 showed higher butyrate levels at the expense of propionate concentrations, in both $\mathrm{PC}$ and DC respectively. The latter confirmed findings obtained in previous studies where repeated administration of lactulose to an in vitro model of the human proximal colon resulted in high butyrate concentrations accompanied with a significantly reduced propionate production $[17,18]$. Butyrate is considered one of the main energy sources for the gut epithelium and elicits high anti-inflammatory potential [44]. In addition, butyrate has been linked with protective effects against colon cancer and obesity-related disorders [45]. The absence of an effect of lactulose on the production of butyrate with donor 1 in the present study can likely be explained by the observation that the microbial pathways for propionate production were heavily enriched. Indeed, the microbial composition of donor 1 was characterized by a lower species richness and evenness as compared to the other donors, with a high abundance of Veillonellaceae, which contains multiple propionate-producing species [46]. Therefore, administration of lactulose likely stimulated the microbial species that were most dominant, resulting in inter-individual differences at metabolic level. This inter-individual response was not examined in previously performed in vitro studies as they mainly focused on the potential beneficial effects of lactulose on a single microbial community.

In-depth analysis of microbial interactions following lactulose administration elucidated the stimulation of specific microbial pathways in the human DC. A rise in abundance of Rikenellaceae was observed after intake of lactulose crystals for all donors tested. In addition, a donor-specific growth of Tannerellaceae in the microbiota obtained from donor 2 could be detected. These findings were mainly attributed to an increase in OTUs related to Alistipes onderdonkii and Parabacteroides distasonis. Aguirre et al. [20] have shown that an oral solution of lactulose stimulated the growth of Alistipes and Parabacteroides species when administered to the colonic microbiota of lean human individuals, confirming the results obtained in the present study. Furthermore, in the DC the total abundance of Lachnospiraceae and Ruminococcaceae increased during the second week of treatment with lactulose as compared to the wash-out period for all donors tested. For donor 1 and 2, this was partly attributed to increased levels of Faecalibacterium prausnitzii. This is in contrast with previous in vitro studies [18], where lactulose fermentation led to a reduction of Faecalibacterium prausnitzii (and to a further extent Ruminococcaceae). However, former in vitro studies exclusively focussed on the human PC, while Faecalibacterium prausnitzii is specifically enhanced in the DC areas [23]. Furthermore, during previous studies lactulose was supplemented for only 5 consecutive days, whereas the current study showed that effects on specific butyrate-producing bacterial groups, such as Faecalibacterium prausnitzii, where only observed during the second application period. Faecalibacterium prausnitzii is a potent butyrate-producing bacterium and a common member of the healthy human gut microbiome. It has been reported to be depleted in the gut microbial community of patients with intestinal disorders such as intestinal bowel syndrome and Crohn's disease [47]. It is considered a potential next-generation probiotic because of its potent anti-inflammatory effects through the inhibition of NF-kB activation, induction of antiinflammatory cytokines such as IL-10 and upregulation of regulatory $\mathrm{T}$ cells [48]. Moreover, lactulose utilization has been reported for Faecalibacterium prausnitzii [49] and Rios-Covian et al. [50] demonstrated enhanced butyrate production by cross-feeding interactions between Faecalibacterium prausnitzii and Bifidobacterium adolescentis upon prebiotic supplementation. In the present study, the strongest butyrogenic effect caused by the fermentation of lactulose was detected for the microbiota of donor 2, which indeed showed the most pronounced growth of Bifidobacterium adolescentis and Faecalibacterium prausnitzii, indicating intensified crossfeeding between these bacterial species.

Finally, some donor-specific changes in microbial community composition were observed following addition of lactulose, especially for donor 2. For instance, a strongly increased abundance of an OTU related to Megasphaera sp. was noted in the PC. This confirms findings of Bothe et al. [18], showing an over 60-fold elevation of Megasphaera species in the human PC after repeated dosing of lactulose crystals in vitro. As Megasphaera species are lactate-utilizing, butyrateproducing microorganisms [51], the stimulation of this bacterial group will probably have contributed to the strong butyrogenic effect observed for donor 2 following lactulose exposure. Additionally, stimulation of Akkermansia muciniphila was observed upon cessation of lactulose treatment for donor 2 in the DC. Akkermansia muciniphila is a mucin-degrading microbe and has recently been related to several health-promoting properties. Schneeberger et al. [52] for instance showed that Akkermansia muciniphila inversely correlates with the onset of inflammation caused by high-fat diet-induced obesity in mice [52]. Its higher abundance upon cessation of lactulose application indicates that lactulose addition probably modulated the gut environment in favor of Akkermansia muciniphila. In vivo, prebiotic compounds typically increase mucin production stimulated by enhanced SCFA production resulting in a bloom of Akkermansia muciniphila [53]. Indeed, Mao et al. [54] showed that lactulose application increased Akkermansia muciniphila abundance in a mouse model in response to rising mucin secretion in the gut. 


\section{Conclusion}

The current investigations show that lactulose administration directly increased the abundance of bifidobacteria and lactobacilli in the PC, yielding elevated levels of acetate and lactate. This subsequently stimulated the predominantly present microbial species, resulting in inter-individual effects at metabolic level. Whereas the microbiota of donor 1 specifically supported propionate production, the microbial communities of donor 2 and 3 showed higher butyrate levels upon lactulose supplementation. Overall, a wide spectrum of propionateand especially butyrate-producing microbes were specifically enhanced in the DC, which is of specific interest as many colonic diseases originate in this colon area.

\section{Acknowledgements}

The work was financially supported by Fresenius-Kabi Austria GmbH.

\section{Abbreviations}

$\mathrm{DC}=$ distal colon; $\mathrm{OTU}=$ operational taxonomic unit; $\mathrm{PC}$ $=$ proximal colon; $\mathrm{qPCR}=$ quantitative polymerase chain reaction; $\mathrm{SCFA}=$ Short-chain fatty acid; SHIME $=$ Simulator of the Human Intestinal Microbial Ecosystem.

\section{Appendix}

Treatment - PC

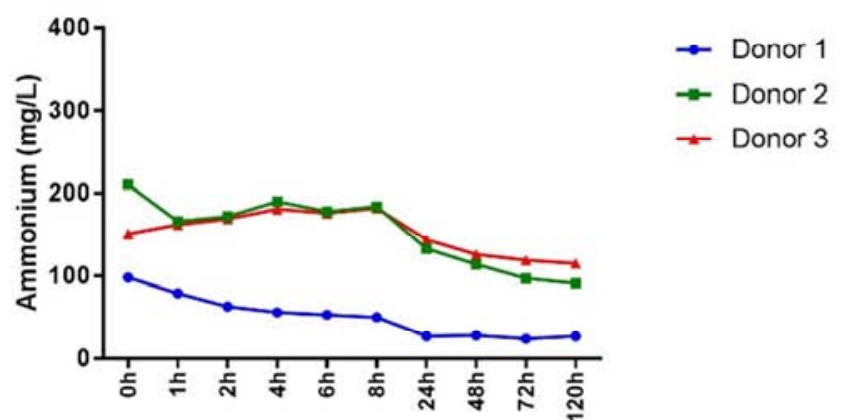

Treatment - DC

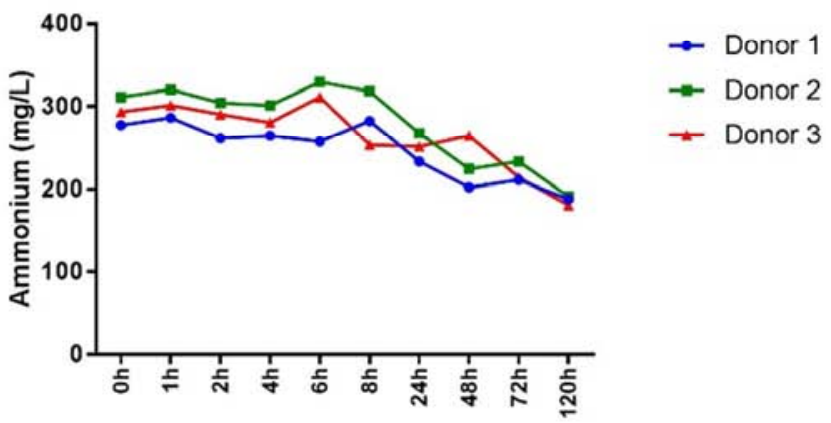

Figure A1. Microbial Metabolic Activity in Terms of Ammonium Production.

Absolute ammonium production ( $\mathrm{mg} / \mathrm{L})$ during the second treatment week with the test product at different time points $(0,1,2,4,6,8,24,48,72$ and $120 \mathrm{~h})$ after the addition of the first feeding to the colon compartments for three different human donors in the proximal (PC) and distal colon (DC).

\begin{tabular}{|c|c|c|c|c|c|c|c|c|c|c|c|c|c|}
\hline \multirow{3}{*}{ Phylum } & \multirow{3}{*}{ Family } & \multicolumn{12}{|l|}{ PC } \\
\hline & & \multicolumn{4}{|c|}{ Donor 1} & \multicolumn{4}{|c|}{ Donor 2} & \multicolumn{4}{|c|}{ Donor 3} \\
\hline & & C & TRl & wo & TR2 & C & TRl & wo & TR2 & C & TRl & wo & TR2 \\
\hline \multirow{2}{*}{ Actinobacteria } & Bifidobacteriaceae & - & - & 0.1 & - & 1.5 & 20.6 & 12.8 & 45.6 & 1.8 & 16.6 & 4.0 & 52.4 \\
\hline & Microbacteriaceae & 0.2 & 0.1 & 0.1 & 0.5 & 0.1 & - & 0.1 & 1.2 & 0.5 & - & - & 3.5 \\
\hline \multirow[t]{2}{*}{ Bacteroidetes } & Bacteroidaceae & 0.9 & - & 3.4 & - & 54.7 & 49.7 & 55.0 & 0.1 & 64.7 & 48.6 & 75.2 & 15.1 \\
\hline & Acidaminococcaceae & - & - & - & - & 5.1 & 9.0 & 4.9 & 0.8 & 4.7 & 5.6 & 4.6 & 4.6 \\
\hline \multirow{3}{*}{ Firmicutes } & Lachnospiraceae & 4.6 & 5.4 & 3.9 & 0.6 & 33.5 & 8.1 & 11.9 & - & 23.2 & 24.4 & 11.6 & 6.6 \\
\hline & Lactobacillaceae & - & - & - & - & 0.3 & 1.9 & 0.8 & 1.0 & - & - & - & - \\
\hline & Veillonellaceae & 93.7 & 94.0 & 92.0 & 94.2 & 1.2 & 8.6 & 10.9 & 39.2 & 0.2 & 2.0 & 1.7 & 3.2 \\
\hline \multirow{5}{*}{ Proteobacteria } & Burkholderiaceae & 0.1 & 0.2 & 0.1 & 0.4 & 1.3 & 1.9 & 3.0 & 4.5 & 1.6 & 2.5 & 1.4 & 3.2 \\
\hline & Desulfovibrionaceae & - & - & - & - & 1.8 & 0.1 & - & 0.1 & 1.9 & - & 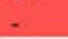 & 0.1 \\
\hline & Enterobacteriaceae & - & - & 0.1 & 3.6 & 0.1 & - & 0.7 & 6.6 & 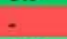 & - & 0.4 & 8.4 \\
\hline & Pseudomonadaceae & 0.1 & 0.1 & 0.1 & 0.2 & 0.2 & - & - & 0.1 & 1.2 & - & 1.0 & 1.4 \\
\hline & Xanthomonadaceae & 0.4 & 0.2 & 0.1 & 0.5 & 0.1 & - & - & 0.5 & 0.2 & - & 0.1 & 1.4 \\
\hline
\end{tabular}

Figure A2. Proximal Community Composition As Assessed Via 16S-targeted Illumina Sequencing at Family Level.

Abundance (\%) at microbial family level in the proximal colon (PC) of the Simulator of the Human Intestinal Microbial Ecosystem (SHIME®) at the end of the control (C), the wash-out (WO) and the two treatment (TR1/2) periods for three different human donors ( $\mathrm{n}=1)$. The intensity of shading indicates the absolute abundance, normalized for each of the different families. Lowest values within each donor are indicated with two shades of red, medium values are indicated in white, whereas the highest values within each donor are indicated with two shades of green. 


\begin{tabular}{|c|c|c|c|c|c|c|c|c|c|c|c|c|c|}
\hline \multirow{3}{*}{ Phylum } & \multirow{3}{*}{ Family } & \multicolumn{12}{|l|}{ DC } \\
\hline & & \multicolumn{4}{|c|}{ Donor 1 } & \multicolumn{4}{|c|}{ Donor 2} & \multicolumn{4}{|c|}{ Donor 3} \\
\hline & & C & TR1 & wo & TR2 & C & TR1 & wo & TR2 & C & TRI & wo & IR2 \\
\hline \multirow{3}{*}{ Actinobacteria } & Bifidobacteriaceae & 0.1 & - & 0.2 & 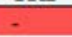 & 1.2 & 12.4 & 8.5 & 9.5 & 0.6 & 4.6 & 1.4 & 7.7 \\
\hline & Coriobacteriaceae & - & - & - & - & - & 0.4 & 0.2 & 0.2 & - & - & 0.1 & 0.1 \\
\hline & Microbacteriaceae & - & - & - & 0.1 & - & - & - & 0.2 & 0.1 & - & & 0.2 \\
\hline \multirow{6}{*}{ Bacteroidetes } & Bacteroidaceae & 13.1 & 2.1 & 7.2 & 6.0 & 38.7 & 19.4 & 23.5 & 26.7 & 34.5 & 51.4 & 60.4 & 39.3 \\
\hline & Marinifilaceae & - & - & - & - & - & - & 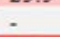 & - & - & 0.1 & 0.1 & 0.2 \\
\hline & Muribaculaceae & 2.9 & 14.8 & 5.5 & 17.1 & 0.8 & 6.3 & 0.7 & 4.4 & - & - & - & - \\
\hline & Prevotellaceae & - & - & - & - & 2.3 & 3.4 & 1.0 & 3.5 & 1.2 & 0.5 & 0.5 & 0.8 \\
\hline & Rikenellaceae & - & 0.8 & 0.2 & 1.2 & 0.2 & 0.5 & 0.2 & 0.3 & 0.2 & 1.0 & 1.6 & 4.4 \\
\hline & Tannerellaceae & 1.3 & 0.9 & 4.3 & 0.5 & 5.5 & 8.1 & 8.5 & 10.4 & 6.5 & 4.1 & 6.5 & 4.3 \\
\hline \multirow{4}{*}{ Firmicutes } & Acidaminococcaceae & 1.5 & 2.3 & 1.0 & 1.5 & 2.5 & 2.3 & 2.1 & 1.8 & 2.3 & 0.7 & 2.4 & 1.2 \\
\hline & Lachnospiraceae & 2.9 & 4.4 & 3.9 & 5.2 & 36.8 & 36.0 & 14.8 & 20.3 & 34.6 & 27.0 & 19.2 & 28.0 \\
\hline & Lactobacillaceae & - & - & - & - & 0.1 & 0.4 & 0.2 & 0.3 & - & - & - & - \\
\hline & Ruminococcaceae & 0.1 & 0.2 & 0.1 & 0.9 & 1.5 & 0.8 & 0.9 & 5.1 & 2.5 & 0.7 & 1.9 & 1.8 \\
\hline \multirow{3}{*}{ Fusobacteria } & Veillonellaceae & 56.6 & 56.7 & 51.8 & 53.8 & 1.5 & 1.5 & 0.5 & 5.3 & 1.1 & 0.7 & 0.1 & 1.1 \\
\hline & Fusobacteriaceae & 0.1 & & 0.3 & - & 5.0 & 3.6 & 3.4 & 1.0 & 7.5 & 6.0 & 0.7 & 5.6 \\
\hline & Burkholderiaceae & 0.2 & 0.2 & 0.3 & 0.1 & 1.0 & 2.0 & 1.1 & 2.0 & 1.4 & 1.4 & 2.1 & 1.6 \\
\hline \multirow{5}{*}{ Proteobacteria } & Desulfovibrionaceae & 0.3 & 0.3 & 0.7 & 0.3 & 1.2 & 1.4 & 1.5 & 2.0 & 1.2 & 1.0 & 1.6 & 1.3 \\
\hline & Enterobacteriaceae & - & - & - & 0.3 & - & - & - & 0.2 & - & - & - & 0.3 \\
\hline & Pseudomonadaceae & 0.2 & - & 0.1 & 0.1 & 0.9 & 0.1 & 0.4 & 0.7 & 1.5 & 0.1 & 0.8 & 1.0 \\
\hline & uncuitured & 0.2 & - & - & & 0.1 & 0.6 & 1.1 & 1.1 & 4.4 & 0.2 & 0.1 & 0.2 \\
\hline & Xanthomonadaceae & - & - & - & - & - & - & - & - & 0.1 & - & - & 0.1 \\
\hline Verrucomicrobia & Akkermansiaceae & 20.2 & 17.2 & 24.6 & 12.7 & 0.4 & 0.4 & 31.2 & 4.8 & 0.2 & 0.1 & 0.1 & 0.4 \\
\hline
\end{tabular}

Figure A3. Distal Community Composition As Assessed Via 16S-targeted Illumina Sequencing at Family Level.

Abundance (\%) at microbial family level in the distal colon (DC) of the Simulator of the Human Intestinal Microbial Ecosystem (SHIME $\mathbb{B}$ ) at the end of the control (C), the wash-out (WO) and the two treatment (TR1/2) periods for three different human donors $(\mathrm{n}=1)$. The intensity of shading indicates the absolute abundance, normalized for each of the different families. Lowest values within each donor are indicated with two shades of red, medium values are indicated in white, whereas the highest values within each donor are indicated with two shades of green.

\section{References}

[1] Gibson, G. R., et al., Expert consensus document: The International Scientific Association for Probiotics and Prebiotics (ISAPP) consensus statement on the definition and scope of prebiotics. Nat Rev Gastroenterol Hepatol, 2017. 14 (8): p. 491-502.

[2] Panesar, P. S. and S. Kumari, Lactulose: production, purification and potential applications. Biotechnol Adv, 2011. 29 (6): p. $940-8$.

[3] Mao, B., et al., In vitro fermentation of lactulose by human gut bacteria. J Agric Food Chem, 2014. 62 (45): p. 10970-7.

[4] Bass, P. and S. Dennis, The laxative effects of lactulose in normal and constipated subjects. J Clin Gastroenterol, 1981. 3 Suppl 1: p. 23-8.

[5] Weber, F. L., Jr., Lactulose and combination therapy of hepatic encephalopathy: the role of the intestinal microflora. Dig Dis, 1996. 14 Suppl 1: p. 53-63.

[6] Brommage, R., et al., Intestinal calcium absorption in rats is stimulated by dietary lactulose and other resistant sugars. J Nutr, 1993. 123 (12): p. 2186-94.

[7] Van den Heuvel, E. G., et al., Lactulose stimulates calcium absorption in postmenopausal women. J Bone Miner Res, 1999. 14 (7): p. 1211-6.

[8] Bouhnik, Y., et al., Lactulose ingestion increases faecal bifidobacterial counts: a randomised double-blind study in healthy humans. Eur J Clin Nutr, 2004. 58 (3): p. 462-6.

[9] Mizota, T., et al., Effects of low dosages of lactulose on the intestinal function of healthy adults. Vol. 57. 2002. 312-315.
[10] Terada, A., et al., Effect of Lactulose on the Composition and Metabolic Activity of the Human Faecal Flora. Microbial Ecology in Health and Disease, 1992. 5 (1): p. 43-50.

[11] Riggio, O., et al., Effect of lactitol and lactulose administration on the fecal flora in cirrhotic patients. J Clin Gastroenterol, 1990. 12 (4): p. 433-6.

[12] De Preter, V., et al., Effect of lactulose and Saccharomyces boulardii administration on the colonic urea-nitrogen metabolism and the bifidobacteria concentration in healthy human subjects. Aliment Pharmacol Ther, 2006. 23 (7): p. 963-74.

[13] Kaur, A., et al., In vitro batch fecal fermentation comparison of gas and short-chain fatty acid production using "slowly fermentable" dietary fibers. J Food Sci, 2011.76 (5): p. H137-42.

[14] Van den Abbeele, P., et al., Arabinoxylo-Oligosaccharides and Inulin Impact Inter-Individual Variation on Microbial Metabolism and Composition, Which Immunomodulates Human Cells. J Agric Food Chem, 2018. 66 (5): p. 1121-1130.

[15] Minekus, M., et al., A computer-controlled system to simulate conditions of the large intestine with peristaltic mixing, water absorption and absorption of fermentation products. Appl Microbiol Biotechnol, 1999. 53 (1): p. 108-14.

[16] Molly, K., M. Vande Woestyne, and W. Verstraete, Development of a 5-step multi-chamber reactor as a simulation of the human intestinal microbial ecosystem. Appl Microbiol Biotechnol, 1993. 39 (2): p. 254-8.

[17] Bothe, M. K., et al., Dose-Dependent Prebiotic Effect of Lactulose in a Computer-Controlled In Vitro Model of the Human Large Intestine. Nutrients, 2017. 9 (7).

[18] Bothe, M., et al., Lactulose Crystals and Liquid Both Show A Dose-Dependent Prebiotic Effect in a Computer-Controlled In Vitro Model of the Human Proximal Colon. J Food Tech Food Chem 1: 103 Abstract RESEARCH ARTICLE Open Access, 2018. 1 (1). 
[19] Venema, K., et al., The Effect of Lactulose on the Composition of the Intestinal Microbiota and Short-chain Fatty Acid Production in Human Volunteers and a Computercontrolled Model of the Proximal Large Intestine. Microbial Ecology in Health and Disease, 2003. 15 (2-3): p. 94-105.

[20] Aguirre, M., et al., In vitro characterization of the impact of different substrates on metabolite production, energy extraction and composition of gut microbiota from lean and obese subjects. PLoS One, 2014.9 (11): p. e113864.

[21] Eckburg, P. B., et al., Diversity of the human intestinal microbial flora. Science, 2005. 308 (5728): p. 1635-8.

[22] Manach, C., et al., Addressing the inter - individual variation in response to consumption of plant food bioactives: Towards a better understanding of their role in healthy aging and cardiometabolic risk reduction. Molecular Nutrition \& Food Research, 2017. 61 (6): p. 1600557.

[23] Van den Abbeele, P., et al., Microbial community development in a dynamic gut model is reproducible, colon region specific, and selective for Bacteroidetes and Clostridium cluster IX. Appl Environ Microbiol, 2010. 76 (15): p. 5237-46.

[24] Van den Abbeele, P., et al., A combination of xylooligosaccharides and a polyphenol blend affect microbial composition and activity in the distal colon exerting immunomodulating properties on human cells. Journal of Functional Foods, 2018. 47: p. 163-171.

[25] Van de Wiele, T., et al., The Simulator of the Human Intestinal Microbial Ecosystem (SHIME®), in The Impact of Food Bioactives on Health: in vitro and ex vivo models, $\mathrm{K}$. Verhoeckx, et al., Editors. 2015, Springer International Publishing: Cham. p. 305-317.

[26] Possemiers, S., et al., PCR-DGGE-based quantification of stability of the microbial community in a simulator of the human intestinal microbial ecosystem. FEMS Microbiol Ecol, 2004. 49 (3): p. 495-507.

[27] De Weirdt, R., et al., Human faecal microbiota display variable patterns of glycerol metabolism. FEMS Microbiology Ecology, 2010. 74 (3): p. 601-611.

[28] Van de Wiele, T., et al., Prebiotic effects of chicory inulin in the simulator of the human intestinal microbial ecosystem. FEMS Microbiol Ecol, 2004. 51 (1): p. 143-53.

[29] Vilchez-Vargas, R., et al., Analysis of the microbial gene landscape and transcriptome for aromatic pollutants and alkane degradation using a novel internally calibrated microarray system. Environ Microbiol, 2013. 15 (4): p. 101639.

[30] Collado, M. C., et al., Intestinal Integrity and Akkermansia muciniphila, a Mucin-Degrading Member of the Intestinal Microbiota Present in Infants, Adults, and the Elderly. Applied and Environmental Microbiology, 2007. 73 (23): p. 77677770 .

[31] Furet, J. P., et al., Comparative assessment of human and farm animal faecal microbiota using real-time quantitative PCR. FEMS Microbiol Ecol, 2009. 68 (3): p. 351-62.

[32] Rinttila, T., et al., Development of an extensive set of $16 \mathrm{~S}$ rDNA-targeted primers for quantification of pathogenic and indigenous bacteria in faecal samples by real-time PCR. J Appl Microbiol, 2004.97 (6): p. 1166-77.
[33] Klindworth, A., et al., Evaluation of general 16S ribosomal RNA gene PCR primers for classical and next-generation sequencing-based diversity studies. Nucleic Acids Res, 2013. 41 (1): p. e1.

[34] Schloss, P. D. and S. L. Westcott, Assessing and improving methods used in operational taxonomic unit-based approaches for $16 \mathrm{~S}$ rRNA gene sequence analysis. Appl Environ Microbiol, 2011. 77 (10): p. 3219-26.

[35] Kozich, J. J., et al., Development of a dual-index sequencing strategy and curation pipeline for analyzing amplicon sequence data on the MiSeq Illumina sequencing platform. Appl Environ Microbiol, 2013. 79 (17): p. 5112-20.

[36] Wang, Q., et al., Naïve Bayesian Classifier for Rapid Assignment of rRNA Sequences into the New Bacterial Taxonomy. Applied and Environmental Microbiology, 2007. 73 (16): p. 5261-5267.

[37] Van Herreweghen, F., et al., In vitro colonisation of the distal colon by Akkermansia muciniphila is largely mucin and $\mathrm{pH}$ dependent. Benef Microbes, 2017. 8 (1): p. 81-96.

[38] Macfarlane, G. T. and G. R. Gibson, Carbohydrate fermentation, energy transduction and gas metabolism in the human large intestine, in Gastrointestinal Microbiology, R. I. Mackie, B. R. White, and R. E. Isaacson, Editors. 1996, Chapman and Hall: London, United Kingdom. p. 269-318.

[39] Duncan, S. H., P. Louis, and H. J. Flint, Lactate-Utilizing Bacteria, Isolated from Human Feces, That Produce Butyrate as a Major Fermentation Product. Applied and Environmental Microbiology, 2004. 70 (10): p. 5810.

[40] Raybaudi-Massilia, R. M., et al., Control of Pathogenic and Spoilage Microorganisms in Fresh-cut Fruits and Fruit Juices by Traditional and Alternative Natural Antimicrobials. Comprehensive Reviews in Food Science and Food Safety, 2009. 8 (3): p. 157-180.

[41] Ballongue, J., C. Schumann, and P. Quignon, Effects of lactulose and lactitol on colonic microflora and enzymatic activity. Scand J Gastroenterol Suppl, 1997. 222: p. 41-4.

[42] Tuohy, K. M., et al., A Human Volunteer Study to Determine the Prebiotic Effects of Lactulose Powder on Human Colonic Microbiota. Microbial Ecology in Health and Disease, 2002. 14 (3): p. 165-173.

[43] Vanhoutte, T., et al., Molecular monitoring of the fecal microbiota of healthy human subjects during administration of lactulose and Saccharomyces boulardii. Appl Environ Microbiol, 2006. 72 (9): p. 5990-7.

[44] Hamer, H. M., et al., Review article: the role of butyrate on colonic function. Alimentary Pharmacology \& Therapeutics, 2008. 27 (2): p. 104-119.

[45] McNabney, S. M. and T. M. Henagan, Short Chain Fatty Acids in the Colon and Peripheral Tissues: A Focus on Butyrate, Colon Cancer, Obesity and Insulin Resistance. Nutrients, 2017. 9 (12).

[46] Flint, H., et al., Links between diet, gut microbiota composition and gut metabolism. Vol. 74. 2014. 1-10.

[47] Sokol, H., et al., Faecalibacterium prausnitzii is an antiinflammatory commensal bacterium identified by gut microbiota analysis of Crohn disease patients. Proc Natl Acad Sci U S A, 2008. 105 (43): p. 16731-6. 
[48] Qiu, X., et al., Faecalibacterium prausnitzii upregulates regulatory $\mathrm{T}$ cells and anti-inflammatory cytokines in treating TNBS-induced colitis. Journal of Crohn's and Colitis, 2013. 7 (11): p. e558-e568.

[49] Cecchini, D. A., et al., Functional Metagenomics Reveals Novel Pathways of Prebiotic Breakdown by Human Gut Bacteria. PLoS ONE, 2013. 8 (9): p. e72766.

[50] Rios-Covian, D., et al., Enhanced butyrate formation by crossfeeding between Faecalibacterium prausnitzii and Bifidobacterium adolescentis. FEMS Microbiology Letters, 2015. 362 (21): p. fnv176-fnv176.

[51] Hashizume, K., et al., Megasphaera elsdenii JCM1772T normalizes hyperlactate production in the large intestine of fructooligosaccharide-fed rats by stimulating butyrate production. J Nutr, 2003. 133 (10): p. 3187-90.
[52] Schneeberger, M., et al., Akkermansia muciniphila inversely correlates with the onset of inflammation, altered adipose tissue metabolism and metabolic disorders during obesity in mice. Sci Rep, 2015. 5: p. 16643.

[53] Van den Abbeele, P., et al., Arabinoxylans and inulin differentially modulate the mucosal and luminal gut microbiota and mucin-degradation in humanized rats. Environmental Microbiology, 2011. 13 (10): p. 2667-2680.

[54] Mao, B., et al., Lactulose Differently Modulates the Composition of Luminal and Mucosal Microbiota in C57BL/6J Mice. Journal of Agricultural and Food Chemistry, 2016. 64 (31): p. 6240-6247. 